\title{
Genetic screening of Fabry patients with ECoTILLING and HRM technology
}

\author{
Caterina Bono ${ }^{1 \dagger}$, Domenico Nuzzo ${ }^{1 * \dagger}$, Giuseppe Albeggiani ${ }^{1}$, Carmela Zizzo ${ }^{1}$, Daniele Francofonte ${ }^{1}$, \\ Francesco lemolo², Enzo Sanzaro ${ }^{2}$ and Giovanni Duro ${ }^{1}$
}

\begin{abstract}
Background: Anderson-Fabry disease (FD) is caused by a deficit of the $\alpha$-galactosidase A enzyme which leads to the accumulation of complex sphingolipids, especially globotriaosylceramide (Gb3), in all the cells of the body, causing the onset of a multi-systemic disease with poor prognosis in adulthood. In this article, we describe two alternative methods for screening the GLA gene which codes for the $\alpha$-galactosidase A enzyme in subjects with probable FD in order to test analysis strategies which include or rely on initial pre-screening.

Findings: We analyzed 740 samples using ECOTILLING, comparing two mismatch-specificendonucleases, CEL I and ENDO-1, while conducting a parallel screening of the same samples using HRM (High Resolution Melting). Afterwards, all samples were subjected to direct sequencing. Overall, we identified 12 different genetic variations: $-10 \mathrm{C}>\mathrm{T},-12 \mathrm{G}>\mathrm{A},-30 \mathrm{G}>\mathrm{A}$, IVS2-76_80del5, D165H, C172Y, IVS4+16A>G, IVS4 +68 A>G, C.718_719delAA, D313Y, IVS622C>T, G395A. This was consistent with the high genetic heterogeneity found in FD patients and carriers. All of the mutations were detected by HRM, whereas $17 \%$ of the mutations were not found by EcoTILLING. The results obtained by ECOTILLING comparing the CEL I and ENDO-1 endonucleases were perfectly overlapping.
\end{abstract}

Conclusion: On the basis of its simplicity, flexibility, repeatability, and sensitivity, we believe thatHRM analysis of the GLA gene is a reliable presequencing screening tool. This method can be applied to any genomic feature to identify known and unknown genetic alterations, and it is ideal for conducting screening and population studies.

Keywords: Anderson-Fabry, haplotype, screening, HRM, ECoTILLING

\section{Introduction}

Fabry disease (FD) is a lysosomal storage disease caused by a congenital error in glycosphingolipid metabolism resulting from the deficient or absent activity of the lysosomal enzyme $\alpha$-galactosidase A [1]. This enzyme is essential for the sphingolipid recycling process that occurs within cells. It is able to remove the galactose bound in the alpha position in complex sphingolipids, allowing their metabolism. The enzyme deficiency causes the interruption of the process of waste product demolition, leading to the intracellular accumulation of complex sphingolipids, especially globotriaosylceramide (Gb3) [2]. The accumulation of these products is the basis of FD symptomology that includes acroparesthesias, angiokeratoma, corneal and

\footnotetext{
* Correspondence: nuzzo@ibim.cnr.it

+ Contributed equally

'National Research Council-Institute of Biomedicine and Molecular Immunology (CNR-IBIM) - Palermo, Italy

Full list of author information is available at the end of the article
}

lenticular opacities, gastrointestinal problems and diseases of the kidney, heart and central nervous system [3-5]. The GLA gene (12 kb) contains seven exons (92 to $291 \mathrm{bp}$ ), is located on the $\mathrm{X}$ chromosome, and encodes a polypeptide of 429 amino acids [6].

In the literature, more than 500 mutations in the GLA gene have been associated with FD [7]. Of these, only $5 \%$ are localized in exons and are able to cause the formation of a truncated protein. Instead, for the most part, they involve missense mutations generating null alleles or changes in specific domains of the protein; others, such as those that occur in splicing sites, can lead to aberrant splicing and can be responsible for altered forms of the enzyme $[8,9]$. Polymorphisms, small deletions, and insertions in the GLA gene have been detected by direct sequencing [10], through single-strand conformation polymorphism analysis [11], and by denaturing high performance liquid chromatography (DHPLC) [12].
C Biomed Central 
However, these investigative techniques may be limited in the analysis of large numbers of samples, so it is therefore necessary to move towards new analytical approaches which include or rely on pre-screening.

In our study, we performed genetic analyses of the GLA gene in 740 subjects with symptoms related to FD using two different approaches to genetic screening. The first was a method based on the enzymatic cleavage of mismatches, known by the acronym EcoTILLING (Targeting Induced Local Lesions in Genomes), originally used for studying the plant genome and adapted to researching human genetic variations [13]. We conducted these analyses using a screening strategy based on pools of 8 samples using two mismatch-specific endonucleases, CEL I (SURVEYOR Mutation Detection Kit-Transgenomic) and ENDO-1 (Serialgenetic). The second method was based on HRM which is able to detect genetic mutations based on the dissociation temperature of the two strands (Tm) and the analysis of the melting curves (LightCycler480, Roche)[14]. We applied these techniques to analyze the coding regions and intron junction regions of the GLA gene in order to investigate mutations in the coding regions and splicing regulatory sites. Finally, to verify the results obtained, each sample was analyzed by direct sequencing.

\section{Materials and methods DNA extraction and quantification}

DNA was extracted by column extraction (GenElute Blood Genomic DNA Kit, Miniprep - Sigma Aldrich) from 740 peripheral blood samples of subjects with clinical manifestations related to FD symptomatology. The study was approved by University Hospital Ethics Committee and written informed consent was obtained from all participants.

The concentration of the DNA was determined by spectrophotometer (Victor X3 - PerkinElmer), and the concentration was adjusted to $100 \mathrm{ng} /$ microliter in TE $(10 \mathrm{mM}$ Tris- $\mathrm{HCl}$ and $1 \mathrm{mM}$ EDTA, $\mathrm{pH}$ 7.4) or water.

\section{Primer design}

Seven pairs of primers were designed for the analyses of the seven target regions containing the seven exons of the GLA gene and the splicing regulatory regions flanking them, and they were obtained using the GeneTools program. The gene sequence was obtained from the site http://www.ensembl.org (ENSG00000102393). The primers used for the EcoTILLING and HRM analyses were identical but for marking with IRDye700 in forward and IRDye800 in reverse (Table 1).

\section{EcoTILLING pooling}

For the simultaneous analysis of a large number of samples, we chose a strategy of pooling, as described by
Till et al. [15]. For a high-throughput screening, the 740 DNA samples were divided into groups of 64 samples each, arranged in $8 \times 8$ grids and then assembled in pools of 8 samples (Figure 1). Each pool was obtained by adding all the samples present in a row or column of the grid, repeating the procedure for the 8 rows and 8 columns of each grid. The 16 pools derived from each grid were arranged in a second plate and subjected to the EcoTILLING method. The presence of the fragments in two pools containing the same sample allowed us to trace the exact location of the sample in the grid and then to identify the subject.

Each PCR was performed in a final volume of $50 \mu \mathrm{l}$ (Hot Start Master Mix - GE Healthcare) with $1 \mu \mathrm{M}$ of primers, $50 \mathrm{ng}$ of DNA. The amplification was performed using the following parameters: $95^{\circ} \mathrm{C}$ for 2 minutes, 40 cycles with $95^{\circ} \mathrm{C}$ for 30 minutes, $58^{\circ} \mathrm{C}$ for 30 minutes, $72^{\circ} \mathrm{C}$ for 1 minute, and a final extension at $72^{\circ} \mathrm{C}$ for 10 minutes.

Mixing 8 different samples ensures the formation of a heteroduplex in the presence of a mutation. The samples thus obtained were denatured and renaturated according to the following parameters: $95^{\circ} \mathrm{C}$ for 2 minutes, followed by a decrease in temperature of $0.5^{\circ} \mathrm{C}$ for 15 minutes, until $45^{\circ} \mathrm{C}$. The heteroduplexes were subjected to enzymatic digestion by the endonucleases CEL I (SURVEYOR Mutation Detection Kit - Transgenomic) and, in parallel, ENDO-1 (Serialgenetics). The reaction was incubated at $42^{\circ} \mathrm{C}$ for 20 minutes and stopped by adding $1.5 \mu$ stop buffer, or at $37^{\circ} \mathrm{C}$ for 40 minutes and stopped with $5 \mu \mathrm{l}$ stop buffer, respectively. All reagents were supplied by the Surveyor kit (SURVEYOR Mutation Detection Kit Transgenomic) and by the ENDO-1 Endonuclease Kit (Serialgenetics).

\section{Electrophoretic Separation}

The fragments were separated by electrophoretic migration (DNAanalyzer 4300 - Licor) on polyacrylamide gel (KB-plus 6.5\% gel-matrix - Licor). The scanning of gels subjected to electrophoresis permits the detection of the presence of mutations and their location in the analyzed fragment, deduced on the basis of the size of the fragments obtained. The gels were analyzed using the GelBuddy program in manual mode.

\section{PCR-High Resolution Melting (HRM)}

Genetic screening for the presence of mutations in the GLA gene was conducted by Real-Time PCR and subsequent HRM (Figure 2). The PCR was carried out on a final volume of $20 \mu \mathrm{l}$, according to the following amplification program: $95^{\circ} \mathrm{C}$ for $10^{\prime}$ minutes, 50 cycles at $95^{\circ} \mathrm{C}$ for 10 minutes, $62^{\circ} \mathrm{C}$ for 15 minutes, $72^{\circ} \mathrm{C}$ for 18 minutes. The amplification in Real-Time PCR and the dissociation of the two DNA strands were performed in the presence of a fluorescent marker (Light Cycler ${ }^{\circledR} 480$ High Resolution 
Table 1 The seven primer pairs used for HRM analyses and the corresponding amplification fragments

\begin{tabular}{|c|c|c|c|c|}
\hline Region & Primers & Amplicon sizes & Exon sizes & $\mathrm{MgCl}_{2}$ conc. \\
\hline Exon 1 & $\begin{array}{l}\text { 5'-TCTTACGTGACTGATTATTGGTCT-3' } \\
\text { 5'-CACACCCAAACACATGGAAA-3' }\end{array}$ & $416 \mathrm{bp}$ & $254 \mathrm{bp}$ & $3.5 \mathrm{mM}$ \\
\hline Exon 2 & $\begin{array}{l}\text { 5'-TGAAATCCCAAGGTGCCTAATA-3' } \\
\text { 5'-GTACAGAAGTGCTTACAGTCC-3' }\end{array}$ & $314 \mathrm{bp}$ & $174 \mathrm{bp}$ & $3.5 \mathrm{mM}$ \\
\hline Exon 3 & $\begin{array}{l}\text { 5'-ACCTGGTGAAGTAACCTT-3' } \\
\text { 5'-CTCAGCTACCATGGCCT-3' }\end{array}$ & $349 \mathrm{bp}$ & $178 \mathrm{bp}$ & $3.5 \mathrm{mM}$ \\
\hline Exon 4 & $\begin{array}{c}\text { 5'-GCTGGAAATTCATTTCTTCCCC-3' } \\
\text { 5'-GGATGGTGAGAAGTGGTTG-3' }\end{array}$ & $285 \mathrm{bp}$ & $91 \mathrm{bp}$ & $3.5 \mathrm{mM}$ \\
\hline Exon 5 & $\begin{array}{c}\text { 5'-AATCTGTAAACTCAAGAGAAGGCTA-3' } \\
\text { 5'-CTTACCTGTATITACCTTGAATG-3' }\end{array}$ & $349 \mathrm{bp}$ & $161 \mathrm{bp}$ & $3.5 \mathrm{mM}$ \\
\hline Exon 6 & $\begin{array}{l}\text { 5'-GATGCTGTGGAAAGTGGTT-3' } \\
\text { 5'-GCCCAAGACAAAGTTGGTAT-3' }\end{array}$ & $355 \mathrm{bp}$ & $198 \mathrm{bp}$ & $3 \mathrm{mM}$ \\
\hline Exon 7 & $\begin{array}{l}\text { 5'-AGAATGAATGCCAAACTAAC-3' } \\
\text { 5'-ATGAGCCACCTAGCCTTG-3' }\end{array}$ & $443 \mathrm{bp}$ & $295 \mathrm{bp}$ & $3.5 \mathrm{mM}$ \\
\hline
\end{tabular}

The primers used in the EcoTILLING protocol were identical and differentiated by their marking with IRDye700 and IRDye800 in forward and reverse, respectively.

Melting Dye). In order to ensure standardization of the method, male subjects were analyzed by adding a wild type DNA counterpart, in a 1:1 volume ratio.

\section{Sequencing}

To test the reliability of the results obtained, all samples were subjected to direct sequencing according to the following program: $94^{\circ} \mathrm{C}$ for 2 minutes, 30 cycles at $96^{\circ}$ C 30 minutes, $58^{\circ} \mathrm{C}$ for 30 minutes, $72^{\circ} \mathrm{C}$ for 1 minute, holding at $4^{\circ} \mathrm{C}$.

\section{Results}

\section{ECOTILLING}

The need to perform simultaneous analyses of many samples led to the adoption of a high-throughput screening strategy based on pools of 8 samples, as reported by Till et al. [15]. In this study, we have carried out genetic screening of the seven chosen target regions for the analysis of the GLA gene on 740 samples (Figure 3). The analysis was done by comparing two enzymes with endonuclease activity (ENDO-1 and CEL I), and their recognition efficiency and heteroduplex cut fragments were identical. The mutations and polymorphisms identified by EcoTILLING are shown in Table 2.

\section{High Resolution Melting}

In order to evaluate the results obtained by EcoTILLING, we conducted another screening with High Resolution Melting. The ten mutations/polymorphisms identified by EcoTILLING were confirmed, and two further gene variations (G395A; IVS6-22c $>$ t), undetected by enzymatic digestion by the CEL I and ENDO-1 endonucleases, were identified (Table 3). The mutations which were undetected by EcoTILLING were analyzed

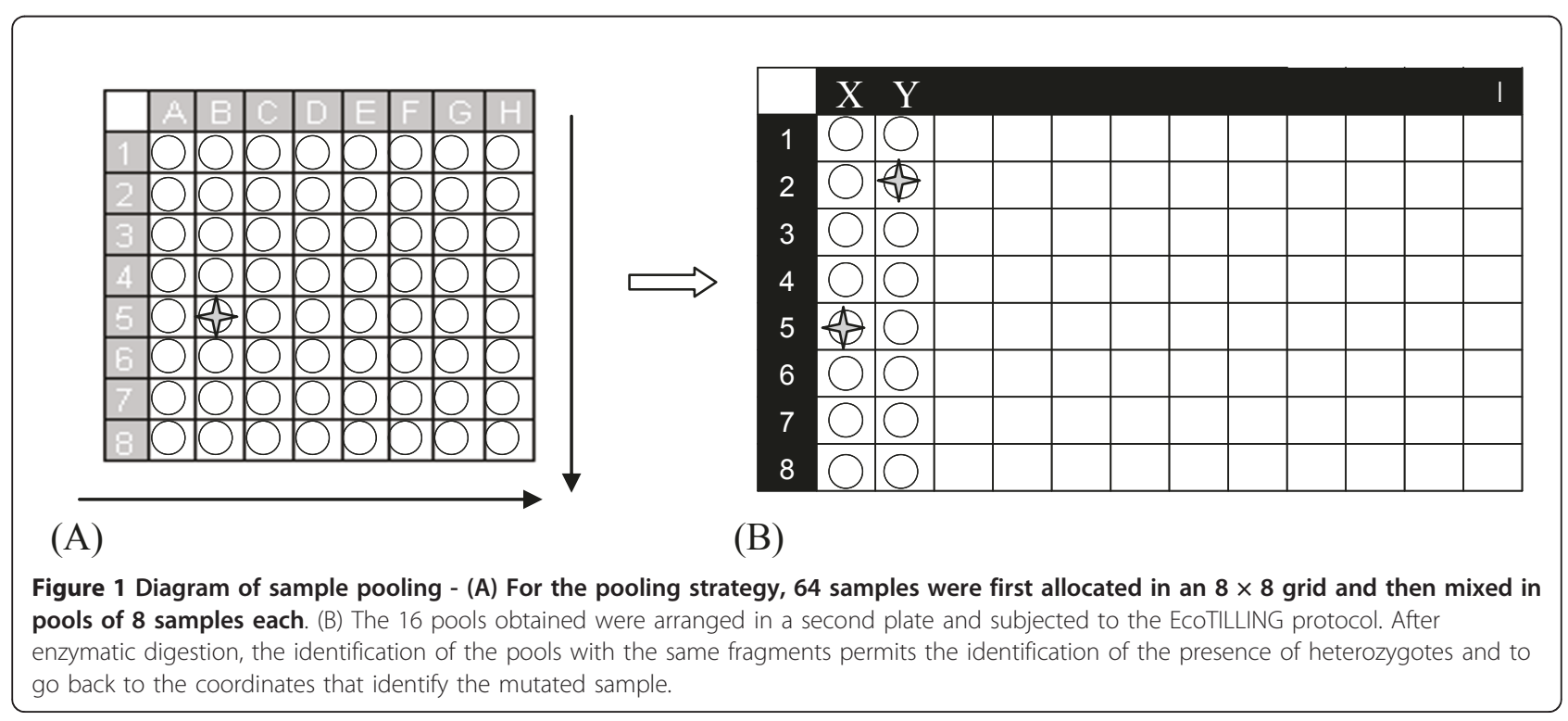



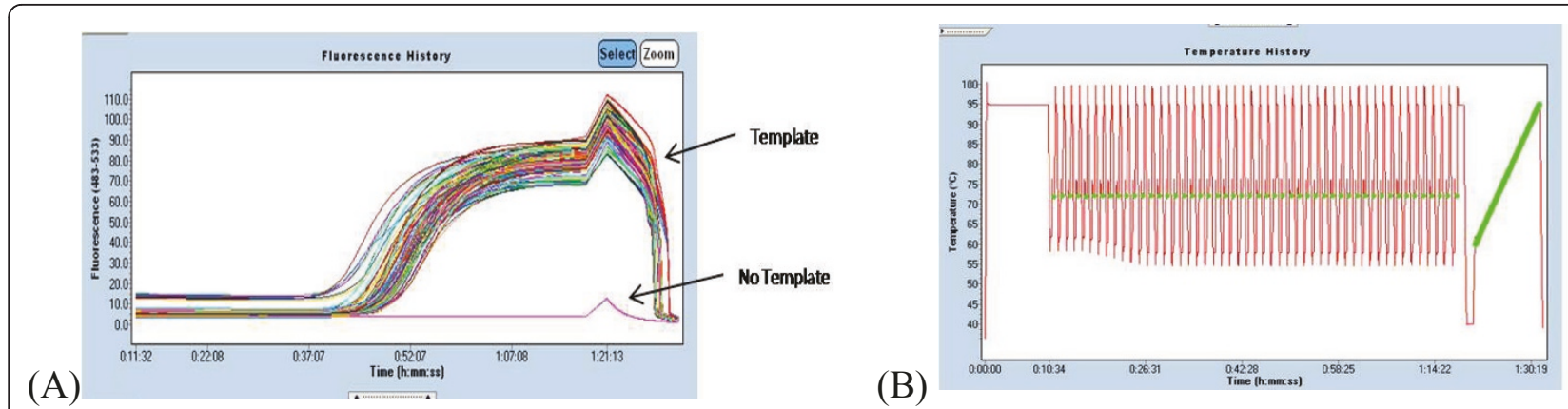

Figure 2 Amplification curves - (A) Amplification curves with characteristic sigmoid pattern. (B) Cycles of amplification, with the moments of data acquisition by the CCD camera indicated in green. The area highlighted by a solid green line represents the dissociation phase of the strands.

separately, applying variations of the protocols: increases in the enzymatic digestion times or changes in analysis of the relationship between mutated and wild type samples in the pool (Figure 4).

After direct sequencing of the 740 samples, no discrepancies were found in the HRM results (Table 4). According to the data, this technique appears to be the optimal method for pre-sequencing screening of the GLA gene. Indeed, the robustness of this method ensures the identification of all possible genomic variants.

\section{Conclusions}

The use of a screening system permits the identification of samples to be subjected to sequencing, the performance of the simultaneous analysis of numerous genetic traits, and the undertaking of population studies.
EcoTILLING technology is recognized by the scientific community as a method for the identification and characterization of single nucleotide polymorphisms (SNPs) and small and large deletions (indels) [16-18]. In this study we analyzed pools of eight samples to reduce screening time and/or the cost associated with the analysis of 740 samples, identifying ten genetic variants across the seven exons and their flanking regions of the GLA gene. After HRM analysis, we discovered two other previously undetected mutations.

The detection of mutations with EcoTILLING shows a number of disadvantages that limit its use in analyses of human genetic diseases and in clinical medicine investigations. In particular, mismatch recognition sensitivity is reduced when mutations are present at the ends of the target sequence $[15,19-23]$ and as the number of polymorphisms identified for each fragment increases.
A)

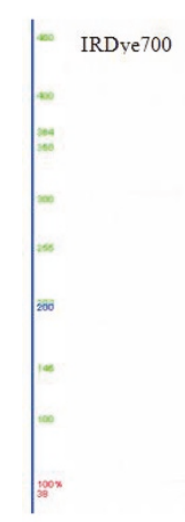

B)

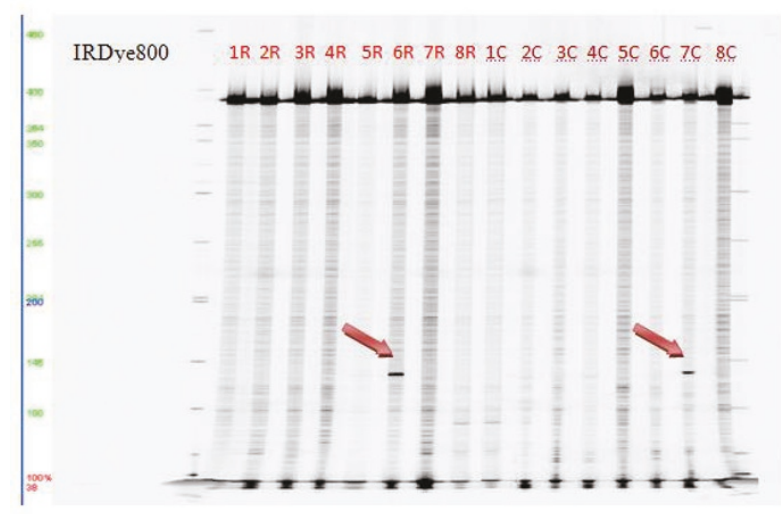

Figure 3 Pooling strategy for Exon 1 of the GLA gene - The 16 lines represent the 16 pools obtained through the pooling strategy for rows (1R-8R) and columns (1C-8C). Above, PCR products undigested at 390bp, visible in both detection channels. (A) Images obtained in the $700 \mathrm{~nm}$ channel for the identification of fragments marked by the forward-IRDye700 primer. (B) Images obtained in the $800 \mathrm{~nm}$ channel for the identification of fragments marked by the reverse-IRDye800 primer. The $6 \mathrm{R}$ and $7 \mathrm{C}$ pools show fragment sizes of approximately 135 bp, indicated by blue arrows (for IRDye 700), and 255 bp, indicated by red arrows (for IRDye 800). Sizes of the digested fragments indicate the position of the mutation within the amplicon, and their sum comprises the dimensions of the undigested 390 bp fragment. 
Table 2 Diagram of the identified genetic variations after enzymatic digestion with CEL I and ENDO-1 and their location

\begin{tabular}{|c|c|c|c|c|c|c|c|c|}
\hline & & Region 1 & Region 2 & Region 3 & Region 4 & Region 5 & Region 6 & Region 7 \\
\hline & & Exon 1 & Exon 2 & Exon 3 & Exon 4 & Exon 5 & Exon 6 & Exon 7 \\
\hline Haplotype 1 & & $-10 \mathrm{C}>\mathrm{T}$ & wt & IVS2 - 76_80del5 & wt & $\begin{array}{c}I V S 4 \\
-16 A>G \\
\end{array}$ & wt & wt \\
\hline Haplotype 2 & Polymorphism & wt & wt & IVS2 - 76_80del5 & wt & $\begin{array}{c}\text { IVS4 } \\
-16 A>G\end{array}$ & wt & wt \\
\hline \multirow[t]{8}{*}{ Haplotype 3} & & $-12 G>A$ & wt & wt & $\begin{array}{c}\text { IVS4 } \\
+68 \mathrm{~A}>\mathrm{G}\end{array}$ & wt & wt & wt \\
\hline & & $-10 C>T$ & wt & wt & wt & wt & wt & wt \\
\hline & Mutations & $-30 G>A$ & wt & wt & wt & wt & wt & wt \\
\hline & & wt & wt & $\mathrm{C} 172 \mathrm{Y}$ & wt & wt & wt & wt \\
\hline & & wt & wt & wt & wt & wt & D313Y & wt \\
\hline & & wt & wt & wt & wt & wt & wt & wt \\
\hline & & wt & wt & wt & wt & c.718_9 dellAA & wt & wt \\
\hline & & wt & wt & $\mathrm{D} 165 \mathrm{H}$ & wt & wt & wt & wt \\
\hline
\end{tabular}

The acquisition of melting profiles in HRM is not dependent on the location and number of mutations present in the analyzed fragment, but only on changes in the dissociation kinetics of the amplified fragments with respect to the wild type [24-27]. Because of its high reliability and flexibility, it is currently employed as a method of genotyping in clinical and diagnostic settings $[28,29]$. In genetics, this method has been applied to BRCA $1 / 2$ hereditary breast cancer [30], cystic fibrosis [31], haemophilia [32], the instability of microsatellites [33], the rearrangement of heavy chain genes in lymphomas [34], the prenatal diagnosis of B-thalassaemia [35], analysis of DNA methylation [36], the identification of bacterial species $[37,38]$, and other applications. Considering the polymorphic nature of the GLA gene, we sustain that HRM analysis appears to be an ideal investigative tool which permits the search for all possible gene variations with a high level of reliability.

Finally, in the cases reported, some mutations seemed to occur in haplotype patterns, as studies of genealogical trees of the proband families confirmed. Indeed, $12 \%$ of the subjects analyzed showed polymorphisms in the promoter region of the GLA gene and, of these, 99\% exhibited several simultaneous polymorphisms spread throughout the gene. In particular, the -10 c >t, IVS2-76_80 del5, IVS4-16 A>G, IVS6-22C >T and -12 g>a, IVS4+68 A>G, IVS6-22C $>\mathrm{T}$ polymorphisms occured simultaneously in $8.9 \%$ and $3.7 \%$ of the subjects, and the significance of this haplotype in FD pathology remains unknown. In a pilot study of patients with small fiber neuropathy of unknown etiology, published in August 2010, Tanislav et al. reported the presence of a complex intronic haplotype within the

Table 3 List of genetic variants found in the samples analyzed by enzymatic digestion using the CEL I or ENDO-1 endonuclease and by High Resolution Melting

\begin{tabular}{|c|c|c|c|}
\hline & Variation & EcoTILLING (Cell or Endol) & HRM \\
\hline \multirow[t]{3}{*}{ Region 1} & $-10 c>t$ & yes & yes \\
\hline & $-12 g>a$ & yes & yes \\
\hline & $-30 \mathrm{~g}>\mathrm{a}$ & yes & yes \\
\hline Region 2 & IVS2-76_80 del5 & yes & yes \\
\hline \multirow[t]{2}{*}{ Region 3} & $\mathrm{D} 165 \mathrm{H}$ & yes & yes \\
\hline & C172Y & yes & yes \\
\hline Region 4 & IVS4+68 A>G & yes & yes \\
\hline \multirow[t]{2}{*}{ Region 5} & IVS4-16 A>G & yes & yes \\
\hline & c.718_719 del AA & yes & yes \\
\hline Region 6 & D313Y & yes & yes \\
\hline \multirow[t]{2}{*}{ Region 7} & IVS6-22 C>T & no & yes \\
\hline & G395A & no & yes \\
\hline
\end{tabular}

The G395A mutation and the IVS6-22C>T polymorphism, identified exclusively through HRM, are shown in bold. 


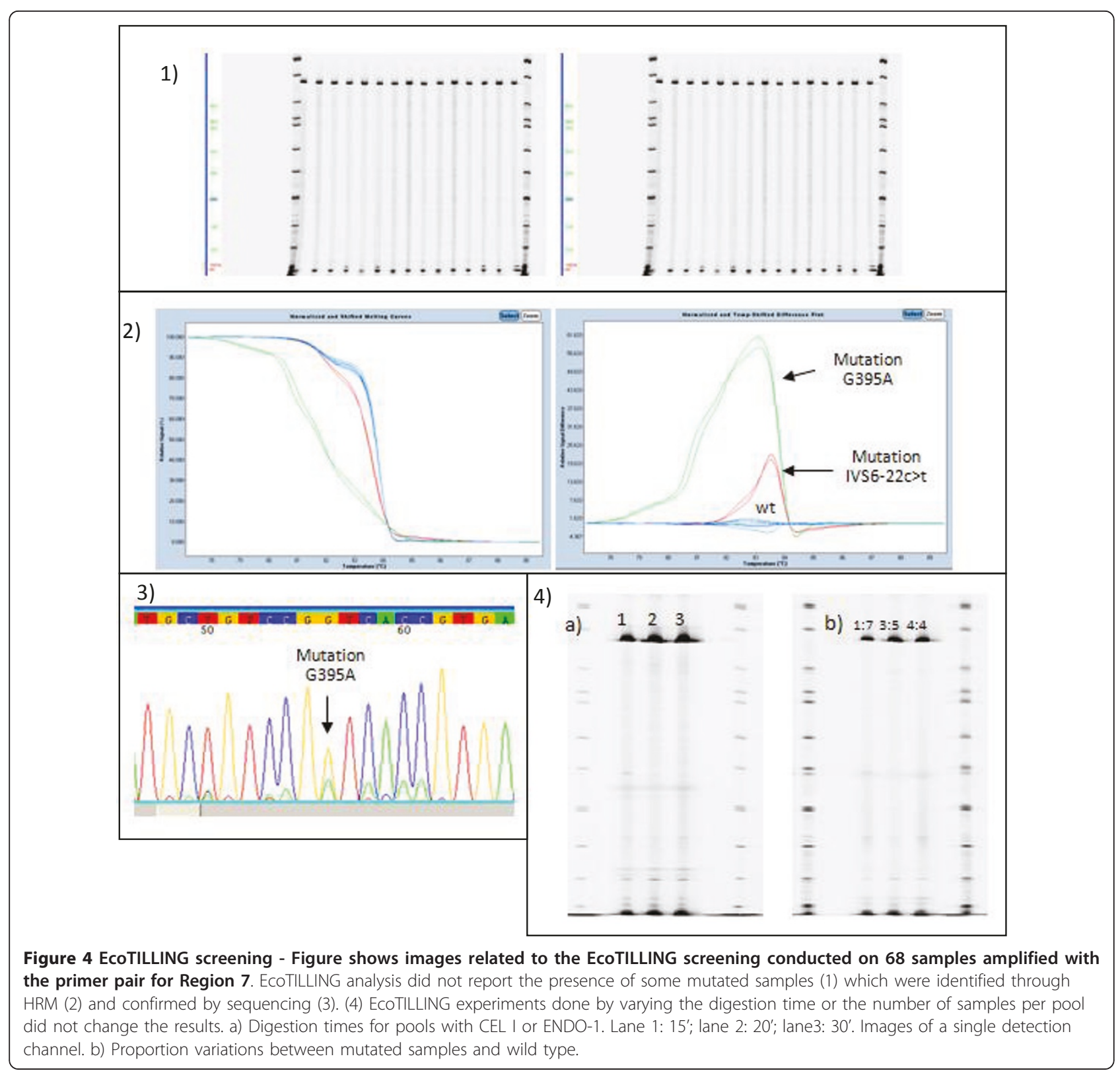

Table 4 Diagram of the genetic variations identified after HRM and their location.

\begin{tabular}{|c|c|c|c|c|c|c|c|c|}
\hline & & Region 1 & Region 2 & Region 3 & Region 4 & Region 5 & Region 6 & Region 7 \\
\hline & & Exon 1 & Exon 2 & Exon 3 & Exon 4 & Exon 5 & Exon 6 & Exon 7 \\
\hline Haplotype 1 & \multirow{4}{*}{ Polymorphism } & $-10 C>T$ & wt & IVS2 - 76_80del5 & wt & IVS4 -16A>G & wt & IVS6 $-22 C>T$ \\
\hline Haplotype 2 & & wt & wt & IVS2 -76_80del5 & wt & IVS4 -16A>G & wt & IVS6 $-22 C>T$ \\
\hline \multirow[t]{8}{*}{ Haplotype 3} & & $-12 \mathrm{G}>\mathrm{A}$ & wt & $w t$ & IVS4 +68A>G & wt & $w t$ & IVS6 $-22 C>T$ \\
\hline & & wt & wt & wt & wt & wt & wt & IVS6 $-22 C>T$ \\
\hline & Mutations & $-30 G>A$ & wt & wt & wt & wt & wt & wt \\
\hline & & wt & wt & C172Y & wt & wt & wt & wt \\
\hline & & wt & wt & wt & wt & wt & D313Y & wt \\
\hline & & wt & wt & wt & wt & wt & wt & G395A \\
\hline & & wt & wt & wt & wt & c.718_9 dellAA & wt & wt \\
\hline & & wt & wt & $\mathrm{D} 165 \mathrm{H}$ & wt & wt & wt & wt \\
\hline
\end{tabular}


GLA gene (-10 c>t, IVS2-76_80 del5, IVS4-16 A>G, IVS6$22 \mathrm{C}>\mathrm{T}$ ) in four patients which is identical to the first of the haplotypes mentioned above for our subjects [39]. The -12 g>a, IVS4+68 A>G, IVS6-22C>T haplotype identified in approximately $4 \%$ of our samples has not been reported in the literature.

\section{Acknowledgements}

This work was made possible by a contribution from Shire Italia. The study was approved by University Hospital Ethics Committee and written informed consent was obtained from all participants.

\section{Author details}

${ }^{1}$ National Research Council-Institute of Biomedicine and Molecular Immunology (CNR-IBIM) - Palermo, Italy. ${ }^{2}$ Department of Neurology Guzzardi Hospital - Vittoria (Ragusa), Italy.

\section{Authors' contributions}

CB drafted the manuscript and conducted ECoTILLING experiments, DN design the study and conducted HRM experiments, GA carried out the molecular genetic study, CZ performed the statistical analysis and participate in the sequence alignment, DF participate in the sequence alignment, $\mathrm{FI}$ participated in acquisitions of a data and interpretations of data, ES participated in acquisitions of a data and interpretations of data, GD participated in the coordination of the study obtaining the informed consent and ethics approval to conduct the study approval. All authors read and approved the final manuscript.

\section{Competing interests}

The authors declare that they have no competing interests.

Received: 1 March 2011 Accepted: 6 September 2011

Published: 6 September 2011

\section{References}

1. Hoffman Bjorn: Fabry disease: recent avances in pathology, diagnosis, treatment and monitoring. Orphanet J Rare Dis 2009, 4:21.

2. Aerts MJohannes, et al: Elevated globotriaosylsphingosine is a hallmark of Fabry disease. Proc Natl Acad Sci USA 2008, 105(8):2812-2817.

3. Shen Jin-Song, et al: Globotriaosylceramide induces oxidative stress and up-regulates cell adhesion molecule expression in Fabry disease endothelial cells. Mol Genet Metab 2008, 95(3):163-168.

4. Linhart Aleš, Elliott MPerry: The heart in AndersonıFabry disease and other lysosomal storage disorders. Heart 2007, 93(4):528-535.

5. Schiffmann Raphael, et al: Fabry disease: progression of nephropathy, and prevalence of cardiac and cerebrovascular events before enzyme replacement therapy. Nephrol Dial Transplant 2009, 24(7):2102-2111.

6. Shows TB, et al: Assignment of a-Galactosidase (aGal) to the q22-qter region of the X chromosome in man. Cytogenet Cell Genet 1978, 22:541-4.

7. Eng CM, et al: Nature and frequency of mutations in the alphagalactosidase A gene that cause Fabry disease. Am J Hum Genet 1993, 53(6):1186-1197.

8. Filoni $C$, et al: Functional studies of new GLA gene mutations leading to conformational fabry disease. Biochimica et Biophysica Acta 2010, 1802:247-252

9. Lin HY, et al: Novel human pathological mutations. Gene symbol: GLA. Disease: Fabry disease. Hum Genet 2010, 127(1):124..

10. Eng CM, et al: Nature and frequency of mutations in the a-galactosidase A gene that cause Fabry disease. Am J Hum Genet 1993, 53:1186-97.

11. Davies JP, et al: Sequence variations in the first exon of a-galactosidase A. J Med Genet 1993, 30:658-63.

12. Shabbeer J, et al: Detection of a-galactosidase a mutations causing Fabry disease by denaturing high performance liquid chromatography. Hum Mutat 2005, 25:299-305.

13. Barkley NA, Wang M: Application of TILLING and EcoTILLING as Reverse Genetic Approaches to Elucidate the Function of Genes in Plants and Animals. Curr Genomics 2008, 9(4):212-26.
14. Montgomery $J$, et al: High-resolution DNA melting analysis in clinical research and diagnostics. Expert Rev Mol Diagn 2010, 10(2):219-40.

15. Till BJ, et al: High-throughput discovery of rare human nucleotide polymorphisms by Ecotilling. Nucleic Acids Res 2006, 34(13):e99.

16. Coassin S, et al: An optimized procedure for the design and evaluation of Ecotilling assays. BMC Genomics 2008, 9:510.

17. Till BJ, et al: High-throughput TILLING for functional genomics. Methods Mol Biol 2003, 236:205-20.

18. McCallum CM, et al: Targeted screening for induced mutations. Nat Biotechnol 2000, 18(4):455-7.

19. Greene EA, et al: Spectrum of chemically induced mutations from a large-scale reversegenetic screen in Arabidopsis. Genetics 2003, 164:731-740.

20. Till BJ, et al: Discovery of chemically induced mutations in rice by TILLING. BMC Plant Biol 2007, 7:19.

21. Raghavan C, et al: Rapid method for detecting SNPs on agarose gels and its application in candidate gene mapping. Mol Breed 2007, 19:87-101.

22. Comai $L$, et al: Efficient discovery of DNA polymorphisms in natural populations by Ecotilling. Plant J 2004, 37:778-786.

23. Gilchrist EJ, et al: Use of Ecotilling as an efficient SNP discovery tool to survey genetic variation in wild populations of Populus trichocarpa. Mol Ecol 2006, 15:1367-1378.

24. Wittwer $C T$, et al: High-resolution genotyping by amplicon melting analysis using LCGreen. Clin Chem 2003, 49:853-860.

25. Erali M, et al: SNP genotyping by unlabeled probe melting analysis. Methods Mol Biol 2008, 429:199-206.

26. Reed $\mathrm{GH}$, et al: Sensitivity and specificity of single-nucleotide polymorphism scanning by high-resolution melting analysis. Clin Chem 2004, 50:1748-1754.

27. Liew $M$, et al: Genotyping of single-nucleotide polymorphisms by highresolution melting of mall amplicons. Clin Chem 2004, 50:1156-1164.

28. Erali Maria, et al: High Resolution Melting Applications for Clinical Laboratory Medicine. Exp Mol Pathol 2008, 85(1):50-58.

29. Krypuy $M$, et al: High resolution melting analysis for the rapid and sensitive detection of mutations in clinical samples: KRAS codon 12 and 13 mutations in non-small cell lung cancer. BMC Cancer 2006, 6:295.

30. Takano EA, et al: Rapid detection of carriers with BRCA1 and BRCA2 mutations using high resolution melting analysis. BMC Cancer 2008, 8:59.

31. Krenková $P$, et al: Evaluation of high-resolution melting (HRM) for mutation scanning of selected exons of the CFTR gene. Folia Biol (Praha) 2009, 55(6):238-42.

32. Laurie $A D$, et al: Detection of factor VIII gene mutations by highresolution melting analysis. Clin Chem 2007, 53(12):2211-4.

33. Janavicius $\mathrm{R}$, et al: Microsatellite instability detection by high-resolution melting analysis. Clin Chem 2010, 56(11):1750-7.

34. Kummalue T, et al: Detection of monoclonal immunoglobulin heavy chain gene rearrangement (FR3) in Thai malignant lymphoma by High Resolution Melting curve analysis. Diagn Pathol 2010, 5:31.

35. Pornprasert S, Sukunthamala K: SYTO9 and SYBR GREEN1 with a highresolution melting analysis for prenatal diagnosis of $\beta$-thalassemia/ hemoglobin-E. Eur J Haematol 2010, 85(5):424-9.

36. Wong EM, Dobrovic A: Assessing gene-specific methylation using HRMbased analysis. Methods Mol Biol 2011, 687:207-17.

37. Castellanos $E_{\text {, et }}$ al: Rapid identification and differentiation of Mycobacterium avium subspecies paratuberculosis types by use of realtime PCR and high-resolution melt analysis of the MAP1506 locus. J Clin Microbiol 2010, 48(4):1474-7.

38. Ong DC, et al: Rapid detection of rifampicin- and isoniazid-resistant Mycobacterium tuberculosis by high-resolution melting analysis. J Clin Microbiol 2010, 48(4):1047-54

39. Tanislav C, et al: Frequency of Fabry disease in patients with small-fibre neuropathy of unknown aetiology: a pilot study. Eur J Neurol 2010.

doi:10.1186/1756-0500-4-323

Cite this article as: Bono et al:: Genetic screening of Fabry patients with EcoTILLING and HRM technology. BMC Research Notes 2011 4:323. 